\title{
INEQUALITIES INVOLVING CIRCULAR, HYPERBOLIC AND EXPONENTIAL FUNCTIONS
}

\author{
Yogesh J. BAGUL
}

Abstract. This paper is aimed at obtaining some new lower and upper bounds for the functions $\cos x, \sin x / x, x / \cosh x$, thus establishing inequalities involving circulr, hyperbolic and exponential functions.

Mathematics subject classification (2010): 26D05, 26D07.

Keywords and phrases: Jordan's inequality, circular, hyperbolic, exponential, lower-upper bound.

\section{REFERENCES}

[1] D. S. Mitrinovic, Analytic Inequalities, Springer-Verlag, New York, Berlin 1970.

[2] Edward Neuman and József SÁndor, Optimal Inequalities For Hyperbolic And Trigonometric Functions, Bulletin of Mathematical Analysis and Applications, vol. 3, Issue 3(2011), pages 177-181.

[3] Feng Qi, Da-Wei Niu, And Bai-Ni Guo, Refinements, Generalizations, and Applications of Jordan's inequality and related problems, Journal of Inequalities and Applications, vol. 2009, Article ID 271923, 52 pages, 2009.

[4] G. D. Anderson, M. K. Vamanamurthy, M. Vuorinen, Conformal Invarients, Inequalities and Quasiconformal maps, John Wiley and Sons, New York, 1997.

[5] L. ZHu, J. Sun, Six new Redheffer-type inequalities for circular and hyperbolic functions, comput. Math. Appl. 56 (2) (2008), pages 522-529.

[6] P. S. Bullen, A Directory of Inequalities, Pitman Monographs and Surveys in Pure and Applied Mathematics, vol. 97, Addison Wesley Longman Limited, Longman, Harlow 1998.

[7] R. KlÉN, M. VisURI AND M. VuORINEN, On Jordan Type Inequalities for Hyperbolic Functions, J. Inequal. and Appl. (2010) 14 pp. (2010) Art. ID 362548.

[8] Yupei Lv, Gendi WANG, Y Umig ChU, A note on Jordan type inequalities for hyperbolic functions, Appl. Math. Lett. 25 (2012) pages 505-508.

[9] CăTĂLIn BARbU AND LAURIAN-IOAN PişCORAn, Jordan type Inequalities using Monotony of Functions, Journal of Mathematical Inequalities, vol. 8, no. 1 (2014), 83-89.

[10] BhAYo, B. A. AND SÁNDOR, On certain old and new trigonometric and hyperbolic inequalities, J. Anal. Math(2015) 41:3.

[11] Zhen-Hang Yang, Yu-Ming Chu, Jordan Type Inequalities for Hyperbolic Functions and Their Applications, Journal of Function Spaces, vol. 2015, Article ID 370979, 4 pages, 2015. 